\title{
Explorando la relación entre evaluación y confianza gubernamental, posición política y participación electoral
}

\author{
Rocío Salas-Lewin ${ }^{1}$ \\ Chantal Clerc ${ }^{2}$ \\ Fabiana Dinamarca ${ }^{3}$
}

Fecha de recepción: 3 de junio de 2019

Fecha de aprobación: 4 de octubre de 2019

Fecha de publicación: 31 de diciembre de 2019

\begin{abstract}
Resumen
La evaluación que los ciudadanos hacen de los gobiernos además de la confianza que depositan en ellos constituyen elementos relevantes de la estabilidad democrática. No obstante, para comprender a cabalidad estos fenómenos, se requiere articularlos con otras actitudes políticas de importancia. Se propone, entonces, un análisis estadístico exploratorio sobre la relación entre evaluación gubernamental, confianza en el gobierno, posición política y participación electoral, mediante conglomeración y correspondencias múltiples. Aquello se realizó en Chile, utilizando datos de la encuesta CEP —Centro de Estudios Públicos- del año 2016. Se concluyó que la posición política y confianza en el gobierno se relacionan con la evaluación de la gestión gubernamental, aunque sin existir un vínculo claro entre esta última y la participación electoral.
\end{abstract}

Palabras clave: Confianza en el gobierno, evaluación gubernamental, intención de voto, participación electoral, posición política.

Licenciada en Sociología, Tesista de Pregrado, Universidad de Chile, Santiago de Chile. Universidad de Chile, Santiago de Chile, Chile. Contacto: rsalaslewin@gmail.com 2 Licenciada en Sociología, Tesista de Pregrado, Universidad de Chile, Santiago de Chile. Universidad de Chile, Santiago de Chile, Chile. Contacto: chantal.clerc.s@gmail.com

3 Licenciada en Sociología, Tesista de Pregrado, Universidad de Chile, Santiago de Chile. Universidad de Chile, Santiago de Chile, Chile. Contacto: f.dinamarcafernandez@gmail.com 


\title{
Exploring the relationship between government evaluation, trust in government, political position, and electoral participation
}

\begin{abstract}
The citizens' evaluation of governments, as well as the trust they place in them, represent important elements of democratic stability. However, in order to fully understand these phenomena, it is necessary to describe them in conjunction with other relevant political attitudes. Thus, an analysis is proposed of the relationship between government evaluation, trust in government, political position and electoral participation, based on an exploratory statistical analysis by conglomeration and multiple correspondence. This was done using Chilean population data from the 2016 CEP survey (Center for Public Studies). The analysis concludes that political position, along with trust in government, contributes to the citizens' evaluation of government management, yet without a clear link between the latter and electoral participation.
\end{abstract}

Key words: electoral participation, government evaluation, intention to vote, political position, trust in government.

\section{Explorando a relação entre avaliação e confiança governamental, posição política e participação eleitoral}

\section{Resumo}

A avaliação que os cidadãos fazem dos governos além da confiança que depositam neles constituem elementos relevantes da estabilidade democrática. Porém, para compreender completamente estes fenômenos, é necessário articulá-los com outras atitudes políticas de importância. Propõe-se, então, uma análise estatística exploratória sobre a relação entre avaliação governamental, confiança no governo, posição política e participação eleitoral, através de conglomeração e correspondências múltiplas. Tal análise foi realizada no Chile, utilizando dados da pesquisa CEP — Centro de Estudos Públicos- do ano 2016. Foi concluído que a posição política e confiança no governo estão relacionadas com a avaliação da gestão governamental, mesmo que sem existir um vínculo claro entre esta última e a participação eleitoral.

Palavras-chave: Confiança no governo, avaliação governamental, intenção de voto, participação eleitoral, posição política. 


\section{Introducción}

El presente artículo identificó distintos perfiles de evaluación de la gestión gubernamental de los chilenos durante el año 2016, para luego determinar el modo en que estos se estructuraron en torno a la confianza en el gobierno y la posición política individual. Además, se determinó cómo aquellos perfiles incidieron en la intención de asistir a votar en las elecciones presidenciales del año 2017. De esta forma, se pretendió identificar perfiles de sujetos que evalúen la gestión gubernamental en diversas áreas, con evaluaciones positivas o negativas, para luego relacionarlos con la confianza en el gobierno, la posición política de los sujetos y su intención de concurrir a las urnas.

El estudio se realizó en Chile con datos de la encuesta del Centro de Estudios Públicos (CEP) de noviembre y diciembre del año 2016 durante el periodo presidencial de Michelle Bachelet. Debe considerarse que la evaluación de los ciudadanos hacia el gobierno se midió en el marco de la administración de la Nueva Mayoría (ex Concertación), coalición chilena de centroizquierda que tradicionalmente ha disputado el campo electoral con el conglomerado político Chile Vamos (ex Alianza) —de orientación política de derecha y centroderecha. Además, la investigación empleó datos recogidos a un año de las elecciones presidenciales de 2017, por lo que se desconocían los candidatos oficiales a la Presidencia, aunque se reconocían las aspiraciones de Sebastián Piñera como probable candidato opositor. Finalmente, en los comicios de 2017, se enfrentaron en el balotaje el actual presidente Sebastián Piñera — como candidato de Chile Vamos- y Alejandro Guillier — como representante de la Nueva Mayoría, sin incluir el partido Demócrata Cristiano.

La investigación se enmarca en un contexto chileno de desconfianza generalizada en las instituciones políticas tradicionales, así como una reducción del interés por la política, la identificación con los partidos y la participación electoral, cuestiones constatadas por diversos sondeos de opinión (Barozet, 2016; Joig- 
nant, Morales \& Fuentes, 2016; Ruiz, 2016; Segovia, 2016). En este contexto, los chilenos se habrían separado crecientemente de las instituciones políticas, de forma que desaparecerían las bases sociales que antes sustentaban a las fuerzas tradicionales (Barozet, 2016; Ruiz, 2016). Así, existiría una ruptura en la relación entre la política institucional - partidos, Congreso, Poder Ejecutivo, entre otras instituciones- y la sociedad, es decir, una ruptura de lo que previamente era la articulación entre sistema partidario y movimientos sociales (Garretón, 2016).

Aquello se explicaría por la existencia de un sentimiento de malestar con las formas de operar del régimen, lo que se expresaría en protestas y diversos mecanismos de movilización social (Joignant et al., 2016). Sin embargo, la situación no derivaría en una crisis de representación o colapso del sistema, sino en lo que se denomina "malestar con la representación": una combinación de desafección, desaprobación y desconfianza política (Joignant et al., 2016).

Este malestar puede convivir con democracias estables, que a corto plazo logran sobrevivir y auto-reproducirse sin necesidad de grandes transformaciones, pues no afecta sustancialmente las percepciones ciudadanas sobre la democracia, aunque a mediano y largo plazo el malestar comienza a generar problemáticas (Joignant et al., 2016). De esta forma, el malestar se constituye como un factor potencial de afectación de la estabilidad democrática.

Así, la relevancia del estudio radica en que este malestar habría derivado en el decaimiento progresivo de la evaluación gubernamental, la confianza en el gobierno y la participación electoral, lo que hace necesario estudiar la interacción entre aquellos indicadores para alcanzar una mayor comprensión del fenómeno de desafección, desaprobación y desconfianza política en vista de que en el futuro se propongan soluciones a esta problemática que, como señalan los autores, incide en la estabilidad y calidad democrática (Joignant et al., 2016). 
Además, en términos teóricos, cabe preguntarse si el fenómeno de malestar político es generalizado o depende del contexto ideológico institucional. La presente investigación permitió indagar sobre la coincidencia entre la posición política de los ciudadanos y su confianza y evaluación hacia un gobierno de centroizquierda. En este sentido, el estudio permitió observar si los reducidos niveles de confianza y la negativa evaluación son transversales a toda posición política individual, o bien si se concentran en aquellas posiciones políticas opositoras al gobierno de turno. De esta forma, la investigación aporta evidencia empírica a la comprobación de modelos teóricos que plantean la existencia de un malestar generalizado -expresado en una mala evaluación y desconfianza gubernamental-. Asimismo, se indagó sobre la posibilidad de que aquel malestar no sea generalizado, sino que esté mayormente representado en la percepción política de ciudadanos con inclinaciones ideológicas opositoras al gobierno vigente.

Por otro lado, considerando que en Chile ha existido una reducción sistemática de la participación electoral (Barozet, 2016; Joignant et al., 2016; Ruiz, 2016; Segovia, 2016), es relevante determinar si la evaluación gubernamental juega un rol que potencia el fenómeno de abstención, es decir, si quienes evalúan negativamente la gestión gubernamental tenderían a disminuir su intención de asistir a votar por futuros gobiernos. De ocurrir aquello, los gobiernos debiesen incorporar a sus políticas medidas que mejoren su evaluación, con el objetivo de elevar la participación para fortalecer la democracia.

Finalmente, la investigación tiene relevancia metodológica al estudiar la relación entre constructos mediante técnicas exploratorias que pueden ser replicadas en futuros estudios de actitudes políticas. Al mismo tiempo, los resultados obtenidos pueden ser de utilidad para la elaboración y fundamentación de futuras investigaciones sobre participación electoral y confianza gubernamental. 


\subsection{Gestión gubernamental y confianza política}

La confianza política se ha definido como la valoración que los ciudadanos otorgan a atributos relevantes de las instituciones centrales del sistema político (Montero, Zmerli \& Newton, 2008). Los sujetos confiarían cuando perciben que el gobierno actuará en pos de los intereses del país, tratando a los ciudadanos de forma igualitaria y justa (Montero et al., 2008). Asimismo, la confianza se generaría cuando las instituciones efectúan lo que un sujeto cree que corresponde (Segovia, Haye, González, Manzi \& Carvacho, 2008).

Newton y Norris (1999) analizan tres concepciones sobre las que se ha entendido la confianza en la institucionalidad política: 1) con foco en emociones personales que predisponen a los sujetos a confiar en las instituciones; 2) centrándose en las experiencias de vida, como la educación y participación social, que generarían reciprocidad y cooperación entre individuos, favoreciendo la confianza interpersonal e institucional; $y$, finalmente, 3) con foco en el desempeño institucional, donde la efectividad gubernamental determinaría la confianza que los ciudadanos depositan en el gobierno.

Según esta última noción, la evaluación del Poder Ejecutivo podría afectar la confianza que los ciudadanos depositan en el gobierno de turno. Así lo señala Morales (2008), al conceptualizar la confianza institucional como "respaldo específico" al desempeño y resultados de las autoridades políticas y sus gobiernos. En este sentido, el negativo desempeño de la gestión gubernamental se traduciría en una falta de confianza en el gobierno.

En la misma línea, Segovia et al. (2008) señalan que la confianza no deriva de los atributos objetivos de una institución, sino de un proceso de construcción de una evaluación en el que intervienen los atributos de la institución, las reacciones subjetivas de quien juzga y las condiciones de la actividad institucional. De esta forma, existirían dos componentes centrales de la confianza en la institucionalidad política: la "capacidad" y "benevolencia" 
percibidas en una institución (Segovia et al., 2008), de modo que la confianza se otorga por la consideración de que una institución puede hacer lo que se le pide en virtud de sus competencias y de que desea sinceramente hacerlo (Segovia et al., 2008). En este sentido, la "capacidad" de gestión esperada del gobierno incidiría en la confianza que en él se deposita.

Asimismo, se ha considerado que la evaluación que los ciudadanos hacen de las competencias, productividad y efectividad gubernamental serían aspectos relevantes de la confianza política que se le confiere a las administraciones (Montero et al., 2008; Segovia et al., 2008). Así, la percepción ciudadana sobre la calidad de las políticas públicas y acciones gubernamentales determinaría la confianza que los sujetos depositan en el gobierno (Segovia et al., 2008).

Con todo, se sostiene como hipótesis que los perfiles relativos a la evaluación de la gestión gubernamental se diferencian por tener distintas percepciones evaluativas en el total de áreas consideradas: delincuencia, crecimiento económico, salud, educación, transporte público, empleo, inflación, control de la corrupción, pobreza y pensiones. Se constituirán, entonces, tres perfiles con evaluación general baja, media y alta de la gestión gubernamental, los que coincidirían con el grado de confianza que los sujetos le confieren al gobierno, de modo que las evaluaciones bajas, medias o altas se reflejarán en una confianza baja, media o alta, respectivamente.

\subsection{Confianza y posición política}

El espectro izquierda-derecha constituye un dispositivo para el entendimiento de la política, permitiendo a los sujetos orientarse en un mundo político complejo y a los gobiernos comunicarse con la ciudadanía (Fuchs \& Klingemann, 1989). Así, el autoposicionamiento en este espectro determina las actitudes políticas de los individuos y sus percepciones acerca de problemáticas específicas, así como la vivencia positiva o negativa de eventos 
y situaciones políticas particulares (Fuchs \& Klingemann, 1989; Inglehart \& Klingemann, 1979).

En este sentido, las políticas públicas y declaraciones del gobierno constituyen una acción que comunica a la ciudadanía la posición política adoptada por la administración, de modo que los sujetos que se autoposicionan en el mismo lugar que el ejecutivo tenderían a experimentar de manera positiva el desempeño gubernamental como situación política y, en consecuencia, a depositarle su confianza al gobierno. Así lo indican Cárdenas, Parra, Picón, Pineda y Rojas (2007), quienes señalan que el nivel de confianza en el gobierno y otras instituciones políticas cambia al segmentar por posición política.

En línea con lo anterior, Zmerli (2006) plantea que la expresión de confianza en las instituciones políticas, como el gobierno, varía según el segmento de la población, pues se relacionaría con el comportamiento político y la orientación política individual. Esto se condice con lo establecido por otros autores, quienes indican que el continuo de posición política izquierda-derecha tendría influencias importantes sobre la confianza en las instituciones políticas, debido a que las creencias y actitudes orientadas, según este espectro, generarían un marco mental para entender y evaluar el mundo político (Kumlin, 2002).

Por otro lado, en Chile, además de las clásicas identificaciones por religión y clase, se ha identificado un tercer clivaje social que articula las preferencias e identificaciones políticas de los individuos: la oposición o apoyo al régimen de Augusto Pinochet, relacionados con la izquierda y derecha respectivamente (Bargsted \& Somma, 2013; Meléndez \& Rovira, 2017). Así, tanto partidos como ciudadanía se identifican en el continuo político de acuerdo con este clivaje, de modo que los sujetos tienden a respaldar a partidos que coincidan con su percepción acerca de la dictadura, al mismo tiempo que los políticos desarrollan estrategias que aseguren el respaldo de sujetos con su misma posición (Bargsted \& Somma, 2013). En este sentido, la fuerte identifica- 
ción política que genera este clivaje en Chile podría determinar que los individuos confíen y evalúen positivamente a aquellos partidos situados en su mismo polo de creencias.

De lo anterior se deriva la segunda hipótesis del estudio que plantea que los sujetos con posiciones políticas afines a la administración del periodo - izquierda y centroizquierda—, tenderán a confiar bastante en el gobierno y a tener una positiva evaluación de su gestión. Por el contrario, sujetos con posiciones políticas contrarias a la administración - derecha y centroderecha-, tenderán a no confiar en el gobierno y a tener una negativa evaluación de su gestión. Por otro lado, se plantea que sujetos autodefinidos como de centro, independientes o sin posición política, tenderán a confiar y evaluar la gestión del gobierno con un nivel intermedio.

\subsection{Gestión gubernamental y participación electoral}

La idea de "votante racional" plantea que los ciudadanos determinan su intención de asistir o no a votar al sopesar los costos y beneficios de su participación, de forma que votarían solo si los beneficios exceden los costos (Foster, 1984). En este sentido, los sujetos que en su experiencia se han sentido favorecidos por determinadas agrupaciones políticas tenderían a ver en el voto una forma de perpetuar sus beneficios cuando las dificultades de asistir a las urnas no son muy elevadas. Asimismo, los individuos con experiencias desfavorables en determinados contextos gubernamentales tenderían a utilizar el voto - en tanto oposición o abstención - como herramienta de castigo hacia las fuerzas políticas que les generaron insatisfacción.

Así, se ha señalado que el voto se ejerce según el desempeño del partido gobernante, la evaluación gubernamental y la situación económica nacional (Morales, 2016). El voto funcionaría como un mecanismo de recompensa o sanción por parte del elector que se ve influenciado por diversos factores que inciden en su percepción sobre el gobierno y su desempeño (Arriagada, 
Navia \& Schuster, 2010). De esta manera, si el votante evalúa de buena forma la gestión gubernamental, sería más probable que acuda a votar con el objetivo de respaldar a un candidato similar al gobierno de turno. Así, los ciudadanos ejercerían un poder de recompensa al entregarle su voto a cierto candidato; o de sanción al abstenerse, votar por un candidato diferente o anular su voto.

En concordancia con ello, Morlino (2007) problematiza los determinantes de una democracia de calidad. Dentro de las dimensiones más relevantes se encuentra la responsabilidad electoral, que puede hacer valer al gobernante frente al elector en función de sus actos (Morlino, 2007), de modo que la acción de asistir a votar se vería influenciada por la aprobación o desaprobación que los ciudadanos hacen de los actos acometidos por los candidatos electos con anterioridad.

De acuerdo con lo anterior, el estudio hipotetiza que sujetos con una evaluación alta de la gestión gubernamental tendrán elevadas intenciones de asistir a votar con el objetivo de respaldar a un gobierno similar. Por el contrario, quienes evalúen de mala forma al gobierno de turno, no tendrán interés de recompensar su desempeño mediante el ejercicio del voto, por lo que existirán menos incentivos para asistir a votar en elecciones.

Sin embargo, de no probarse esta hipótesis se podría aventurar la idea de un "votante irracional", siguiendo teorías que señalan que el voto sería utilizado como forma de sanción hacia los gobiernos por hechos que no les son imputables - como desastres naturales-, cuestión que se debería principalmente a la desinformación y el desinterés en la política de la ciudadanía (Achen \& Bartels, 2016).

\section{Metodología}

El estudio realizó un análisis de conglomerados para identificar los perfiles de sujetos en torno a la evaluación de la gestión 
gubernamental en distintas áreas públicamente relevantes. Posteriormente, se efectuó un análisis de correspondencias múltiples para determinar cómo se estructuran aquellos perfiles de acuerdo con los niveles de confianza en el gobierno y la posición política de los chilenos. Finalmente, se cruzaron los perfiles de evaluación de la gestión gubernamental con la intencionalidad de asistir a votar en las elecciones presidenciales de 2017, mediante una tabla de contingencia, para determinar cómo se relacionan estas variables.

Para ello se utilizó la base de datos del Estudio Nacional de Opinión Pública $N^{o} 48$ del Centro de Estudios Públicos (CEP), cuyos datos se recogieron durante noviembre y diciembre del año 2016. La población contemplada corresponde a todos los chilenos de 18 años o más, habitantes del país, excluyendo Isla de Pascua, utilizando como marco muestral al Censo de 2002 (CEP, 2016b). La muestra fue de tipo probabilística, por conglomerados en tres etapas - manzanas, viviendas y personas-, seleccionando a un total de 1,872 sujetos, de entre los que se concluyeron 1,464 encuestas (CEP, 2016b). La encuesta fue realizada cara a cara en los hogares seleccionados (CEP, 2016b).

Se utilizó una base de datos con tratamiento listwise, en donde los valores "no sabe" y "no contesta", de todas las variables utilizadas, se dieron por perdidos, eliminándose todos los casos cuya respuesta en alguna de las variables se consideró inválida. Con ello, la muestra original se redujo de 1.464 casos a 1.216, lo que no implicó una mayor pérdida de información para la realización del análisis.

Cabe señalar que, al utilizar datos secundarios de una encuesta de opinión pública, el análisis empleó la variable de intención de voto, no así el voto efectivo. Esto se asocia a dos problemáticas que deben considerarse a la hora de leer los resultados: 1) la conducta electoral no es autoobservable, es decir, los individuos no necesariamente son capaces de anticipar su participación electoral futura, pues ella depende de múltiples factores que confluyen 
durante la campaña y día de la elección, mientras que la encuesta es aplicada con bastante anticipación; y 2) la pregunta que determina la intención de asistir a votar se ve sesgada producto de la deseabilidad social, pues existen exigencias sociales que hacen deseable la participación electoral, produciéndose una sobredeclaración de la motivación de asistir a votar en elecciones futuras (Cumsille \& Nogales, 2014).

\section{Resultados}

\subsection{Identificación de perfiles de evaluación de la gestión gubernamental}

En primera instancia, se realizó un análisis de conglomerados para identificar los perfiles de sujetos en torno a la evaluación de la gestión gubernamental. Para ello, se utilizaron diez variables, correspondientes a la evaluación de la gestión gubernamental en diez distintas áreas: delincuencia, crecimiento económico, salud, educación, transporte público, empleo, inflación, control de la corrupción, pobreza y pensiones. La pregunta señalaba: "Entre 1 y 7, ¿Qué nota le pondría usted al gobierno por su gestión en...?", siendo las categorías de respuesta los valores discretos de uno a siete (CEP, 2016b). La muestra utilizada es mayor a 200 casos, por lo que se empleó el método de conglomeración no jerárquico, que permite el tratamiento de muestras grandes (Cea, 2004).

Los coeficientes de asimetría de las variables se encuentran dentro del intervalo \pm 2 , lo que permite utilizar la matriz de correlación de Pearson para evaluar la colinealidad de las variables analizadas. Los resultados indican que la asociación entre variables oscila entre .375 - para las variables de evaluación de la gestión gubernamental en transporte y delincuencia- y .687 - para las variables de evaluación de la gestión gubernamental en empleo y crecimiento. Así, existe una baja colinealidad, lo que indica que todas las variables utilizadas contribuyen a 
la conglomeración, de modo que ninguna podría ser excluida del modelo al estar siendo suplida por una variable a la que se asocia (Cea, 2004). Así, todas las variables utilizadas son estadísticamente relevantes y deben permanecer en el modelo de conglomeración.

Para la utilización del método de conglomeración no jerárquico deben definirse a priori la cantidad de conglomerados a utilizar. Se recomienda establecer dos a cinco conglomerados, sin embargo, un modelo de dos conglomerados tendría baja homogeneidad interna, contrario a los supuestos del análisis (Cea, 2004). Por otro lado, un modelo de cinco o cuatro conglomerados dificulta la interpretación, pues existe multiplicidad de agrupaciones, lo que no se condice con el principio de parsimonia, que indica como positiva la simplicidad del modelo para un análisis e interpretación más sencillo (Cea, 2004). Así, se definió realizar un modelo de tres conglomerados, de modo que exista heterogeneidad entre ellos y homogeneidad interna, a la vez que se cumple el principio de parsimonia.

Ante lo anterior, se puede señalar que un modelo de tres conglomerados permite identificar perfiles mayormente definidos, en concordancia con la hipótesis de investigación que plantea que existe una evaluación general de la gestión gubernamental diferenciada en cada perfil como baja, media y alta.

El modelo de conglomeración se ejecutó excluyendo los casos atípicos multivariantes, pues estos perjudicarían la homogeneidad al interior de los conglomerados, siendo recomendable eliminarlos en muestras grandes (Cea, 2004). Con ello, la muestra se redujo a 1.181 casos, cuestión que no excede el 3\% de la muestra listwise inicial. En este sentido, se justifica su eliminación, pues no se pierden grandes cantidades de información relevante para el análisis (Cea, 2004).

La siguiente tabla presenta los resultados del modelo de conglomeración: 
Tabla 1

Medias de evaluación de la gestión gubernamental - modelo de tres conglomerados

\begin{tabular}{|l|c|c|c|}
\hline & \multicolumn{3}{|c|}{ Conglomerados } \\
\hline & 1 & 2 & 3 \\
\hline Delincuencia & 1.32 & 2.09 & 3.42 \\
\hline Crecimiento & 1.98 & 3.43 & 4.48 \\
\hline Salud & 1.75 & 3.08 & 4.39 \\
\hline Educación & 1.92 & 3.60 & 4.60 \\
\hline Transporte & 2.07 & 3.54 & 4.48 \\
\hline Empleo & 1.89 & 3.46 & 4.49 \\
\hline Inflación & 1.86 & 3.39 & 4.36 \\
\hline Corrupción & 1.43 & 2.29 & 3.82 \\
\hline Pobreza & 1.62 & 3.03 & 4.27 \\
\hline Pensiones & 1.52 & 2.43 & 3.87 \\
\hline Tamaño & 367 & 478 & 336 \\
\hline Nota: Elaboración propia a partir de: Centro de Estudios Públicos (2016a). \\
\hline
\end{tabular}

Se observa que el modelo obtiene una cantidad de casos por conglomerado bastante similar. Además, se pueden revisar las medias obtenidas en cada conglomerado por variable, a modo de caracterizarlos. Se aprecia que se constituyen tres perfiles de sujetos de acuerdo con su evaluación de la gestión gubernamental en distintas áreas. El primer perfil obtiene promedios de evaluación de la gestión gubernamental que rondan el uno en una escala de 1 a 7, siendo esta una evaluación baja. Por su parte, el segundo y tercer perfil obtienen promedios medios de evaluación de la gestión en distintos ámbitos, aunque existe una diferencia importante, en tanto el segundo obtiene un promedio de notas alrededor de tres en una escala de 1 a 7 , mientras que el tercero obtiene promedios en torno al cuatro. De este modo, el segundo perfil se caracteriza por tener un promedio de evaluación de la gestión gubernamental medio bajo, mientras que el tercero se caracteriza por promedios medios altos. 
Tabla 2

Perfiles de evaluación de la gestión gubernamental

\begin{tabular}{|l|l|c|}
\hline \multicolumn{1}{|c|}{ Perfil 1 } & \multicolumn{1}{|c|}{ Perfil 2 } & \multicolumn{1}{c|}{ Perfil 3 } \\
\hline $\begin{array}{l}\text { Evaluación de gestión } \\
\text { gubernamental baja. }\end{array}$ & $\begin{array}{l}\text { Evaluación de gestión gu- } \\
\text { bernamental media baja. }\end{array}$ & $\begin{array}{l}\text { Evaluación de gestión gu- } \\
\text { bernamental media alta. }\end{array}$ \\
\hline \multicolumn{2}{|l}{ Nota: Elaboración propia. }
\end{tabular}

Los conglomerados se encuentran claramente diferenciados de modo que existe heterogeneidad entre grupos, cumpliendo aquella condición para el análisis (Cea, 2004). Esto se constata al observar las distancias euclídeas entre conglomerados, todas mayores a 3.8, lo que indica distancias relativamente altas entre ellos, considerando que 0 es distancia nula (Cea, 2004). Como complemento, se analizó el valor F y su significación en las pruebas ANOVA para la diferencia de medias de cada variable según conglomerado, donde valores elevados y significativos implican que las medias de las variables difieren entre conglomerados (Cea, 2004). Los resultados a continuación sugieren valores $\mathrm{F}$ altos — superiores a 431.1- y significativos, al 95\% de confianza, por lo que las medias de todas las variables analizadas se encuentran diferenciadas entre conglomerados, caracterizándolos correctamente y aportando a su construcción.

Tabla 3

Valores F de las variables de conglomeración

\begin{tabular}{|l|c|}
\hline Variable & Valor F \\
\hline Delincuencia & $438.6^{*}$ \\
\hline Crecimiento & $657.2^{*}$ \\
\hline Salud & $658.7^{*}$ \\
\hline Educación & $645.5^{*}$ \\
\hline Transporte & $431.1^{*}$ \\
\hline Empleo & $736.5^{*}$ \\
\hline Inflación & $649.9^{*}$ \\
\hline Corrupción & $529.3^{*}$ \\
\hline Pobreza & $783.1^{*}$ \\
\hline Pensiones & $455.2^{*}$ \\
\hline $\begin{array}{l}\left.\text { Nota: }{ }^{*}\right] \text { p - valor }<0.05 . \text { Elaboración propia a partir de: Centro de Estu- } \\
\text { dios Públicos }(2016 a) .\end{array}$ \\
\hline
\end{tabular}


Ahora bien, en términos globales, es necesario destacar que, a pesar de la distinción entre perfiles de evaluación de la gestión gubernamental, todos evidencian que sus medias más bajas son en la variable delincuencia. En ese sentido, es posible señalar que la delincuencia es un tema transversal que preocupa a la totalidad de la ciudadanía. Por otro lado, la gestión en educación es evaluada con las medias más altas en el segundo y tercer perfil, mientras que la gestión en transporte público obtiene la mejor nota en el primer perfil.

El último punto que destacar es que, si bien se identifica un perfil de evaluación de la gestión gubernamental media alta, sus valores no exceden el 4.8. Esto implica que, en general, no existe una buena evaluación respecto a la gestión gubernamental en sus diversos ámbitos. Pese a ello, se comprueba la hipótesis relativa a que se establecen perfiles de evaluación de la gestión gubernamental diferenciados por la percepción de la gestión en el total de las áreas consideradas: delincuencia, crecimiento económico, salud, educación, transporte público, empleo, inflación, control de la corrupción, pobreza y pensiones, constituyéndose perfiles con una evaluación general baja y media, y no se obtienen perfiles con evaluaciones altas, aunque las evaluaciones medias se diferencien de forma relevante entre media baja y media alta.

Lo anterior se condice con la idea de que existe un malestar generalizado con la institucionalidad, en este caso expresado en la representación gubernamental (Joignant et al., 2016), pues no existiría una gran proporción de sujetos que constituyan un perfil de alta evaluación hacia la gestión del gobierno.

\subsection{Relación entre evaluación gubernamental, confianza en el gobierno y posición política}

A partir de los perfiles obtenidos respecto a la evaluación de la gestión en el análisis de conglomerados, se realizó un análisis de correspondencias múltiples para determinar su estructuración en función de la variable confianza en el gobierno y posición política. 
Así, los perfiles de evaluación de la gestión gubernamental obtenidos mediante el análisis de conglomerados se constituyeron como una nueva variable, de categorías baja, media baja y media alta.

La variable confianza en el gobierno, por su parte, respondía a la pregunta: “¿Cuánta confianza tiene Ud. en el gobierno?” Siendo sus categorías de respuesta: mucha confianza, bastante confianza, poca confianza y nada de confianza (CEP, 2016b). La variable posición política respondía a la pregunta: "En esta tarjeta representamos las distintas posiciones políticas. Por favor, indíqueme, ¿con cuál Ud. se identifica más o con cuál posición simpatiza Ud. más?" Siendo sus categorías de respuesta: derecha, centro derecha, centro, centro izquierda, izquierda, independiente y ninguno (CEP, 2016b).

Se utilizó un mapa de correspondencias simétrico, en donde se muestran simultáneamente los perfiles fila y columna de las variables. Allí, en una tabla, las filas corresponden a observaciones, mientras que las columnas a las variables, de modo que las coordenadas de los puntos del mapa representan los perfiles fila y columna (Greenacre, 2008).

La decisión respecto a cuántas dimensiones se consideró en el análisis se tomó a partir de la tabla de inercias principales expuesta a continuación:

Tabla 4

Inercias principales

\begin{tabular}{|c|c|c|c|}
\hline Dimensión & Valor & $\begin{array}{c}\text { \% de varianza } \\
\text { explicada }\end{array}$ & \% acumulado de varianza explicada \\
\hline 1 & 0.067 & 75.7 & 75.7 \\
\hline 2 & 0.009 & 10.2 & 85.9 \\
\hline 3 & 0.001 & 1.7 & 87.5 \\
\hline 4 & 0.000 & 0.2 & 87.7 \\
\hline \multicolumn{2}{|c|}{ Nota: Elaboración propia a partir de: Centro de Estudios Públicos (2016a). } \\
\hline
\end{tabular}

Se observa en la tabla anterior, según la varianza acumulada, que solo la primera dimensión explica un $75.7 \%$ de la varianza de las variables utilizadas. El empleo de dos dimensiones explicaría un $85.9 \%$ de la varianza de las variables, siendo un $50 \%$ 
el porcentaje ideal de varianza que debiesen explicar el total de dimensiones a utilizar. Así, las primeras dos dimensiones conservan por sí solas la mayor parte de información del modelo. La tercera dimensión no llega a explicar un $2 \%$ de la varianza, lo que disminuye de forma importante hacia la cuarta y quinta dimensión. En este sentido, los porcentajes de varianza acumulada recomiendan la selección de dos dimensiones para el análisis.

Debe señalarse que las dos dimensiones constituyen un modelo de alto porcentaje explicativo, pues superan el 50\% de varianza explicada que es ideal en ciencias sociales. Por otro lado, el empleo de dos dimensiones simplifica la interpretación del mapa perceptual, generando un esquema visualmente más claro, al tiempo que facilita la interpretación sociológica.

Las categorías que más contribuyen a la formación de cada una de las dimensiones del modelo y las variables que más se asocian a ellas, se presentan a continuación:

Tabla 5

Correlaciones cuadráticas y contribuciones a las dimensiones del análisis de correspondencias múltiples

\begin{tabular}{|c|c|c|c|c|c|}
\hline \multirow{2}{*}{ Variables } & \multirow{2}{*}{ Categorías } & \multicolumn{2}{|c|}{ Dimensión 1} & \multicolumn{2}{|c|}{ Dimensión 2} \\
\hline & & cor & ctr & cor & ctr \\
\hline \multirow{7}{*}{ Posición política } & Derecha & 0.779 & 17 & 0.007 & 1 \\
\hline & Centroderecha & 0.034 & 0 & 0.596 & 19 \\
\hline & Centro & 0.865 & 20 & 0.07 & 12 \\
\hline & Centroizquierda & 1 & 70 & 0 & 12 \\
\hline & Izquierda & 0.929 & 84 & 0.013 & 9 \\
\hline & Independiente & 0 & 0 & 0.719 & 80 \\
\hline & Ninguna & 1 & 39 & 0 & 0 \\
\hline \multirow{4}{*}{$\begin{array}{l}\text { Confianza en el } \\
\text { gobierno }\end{array}$} & Mucha confianza & 0.672 & 80 & 0.095 & 85 \\
\hline & Bastante confianza & 0.769 & 154 & 0.058 & 86 \\
\hline & Poca confianza & 0.397 & 31 & 0.375 & 219 \\
\hline & Nada de confianza & 0.784 & 158 & 0.052 & 79 \\
\hline \multirow{3}{*}{$\begin{array}{l}\text { Evaluación de la } \\
\text { gestión guberna- } \\
\text { mental }\end{array}$} & Media alta & 0.875 & 188 & 0.026 & 42 \\
\hline & Media baja & 0.006 & 0 & 0.722 & 232 \\
\hline & Baja & 0.779 & 158 & 0.083 & 126 \\
\hline
\end{tabular}


Para describir las categorías que contribuyen a la formación de cada una de las dimensiones se utilizaron los valores obtenidos por el coeficiente de contribución (ctr). Así, se empleó el criterio convencional, que consiste en dividir 1 por el total de categorías -14 en este caso- y multiplicarlo por 1.000. Esto da como resultado 71.4, de modo que aquellas categorías que se encuentren por sobre este valor contribuyen a la formación de la dimensión.

Como se observa en la tabla anterior, la primera dimensión se compone de las categorías: a) Posición política: izquierda; b) Confianza en el gobierno: bastante confianza y nada de confianza; y c) Evaluación de la gestión gubernamental: baja y media alta. Estas categorías son las que más contribuyen a la formación de la primera dimensión, considerando valores de contribución superiores a 84 . El resto de las categorías que contribuyen con valores superiores a 71.4 obtienen una contribución mayor en la segunda dimensión, de modo que fueron consideradas en lo que sigue. La segunda dimensión, por su parte, se compone de las categorías: a) Posición política: independiente; b) Confianza en el gobierno: mucha confianza y poca confianza; y c) Evaluación de la gestión gubernamental: media baja. Estas categorías son las que más contribuyen a la formación de la segunda dimensión. Aquello, considerando valores de contribución superiores a 80.

Sumado a lo anterior, se debe analizar la intensidad de la asociación de cada categoría con las dimensiones seleccionadas para conocer las categorías que se encuentran mejor explicadas por cada dimensión. Para esto, se observaron los valores de correlación al cuadrado (cor) en la tabla anterior. Estos valores deben indicar una asociación superior a .7, lo que señala una correlación de buena intensidad.

De este modo, se contempló que la primera dimensión mantiene una intensidad de asociación buena con las categorías: a) Posición política: derecha, centro, centroizquierda, izquierda y ninguna; b) Confianza en el gobierno: bastante confianza y nada 
de confianza; y c) Evaluación de la gestión gubernamental: baja y media alta. Los valores de intensidad de asociación de estas categorías con la primera dimensión son superiores a .769. La segunda dimensión, por su parte, mantiene una intensidad de asociación buena con las categorías: a) Posición política: independiente; y b) Evaluación de la gestión gubernamental: media baja. Los valores de intensidad de asociación de estas categorías con la segunda dimensión son superiores a .719.

La siguiente tabla presenta las masas y coeficientes de calidad de la totalidad de categorías sobre el modelo de dos dimensiones:

Tabla 6

Masas y coeficientes de calidad de las categorías en el análisis de correspondencias múltiples

\begin{tabular}{|l|l|c|c|}
\hline Variables & Categorías & Masa & Calidad \\
\hline \multirow{5}{*}{ Posición política } & Derecha & 25 & 786 \\
\cline { 2 - 4 } & Centroderecha & 11 & 631 \\
\cline { 2 - 4 } & Centro & 27 & 936 \\
\cline { 2 - 4 } & Centroizquierda & 21 & 1,000 \\
\cline { 2 - 4 } & Izquierda & 31 & 942 \\
\cline { 2 - 4 } & Independiente & 9 & 719 \\
\cline { 2 - 4 } & Ninguna & 210 & 1,000 \\
\hline \multirow{5}{*}{ Confianza en el gobierno } & Mucha confianza & 5 & 767 \\
\cline { 2 - 4 } & Bastante confianza & 29 & 827 \\
\cline { 2 - 4 } & Poca confianza & 148 & 772 \\
\cline { 2 - 4 } & Nada de confianza & 151 & 837 \\
\hline \multirow{3}{*}{$\begin{array}{l}\text { Evaluación de la gestión } \\
\text { gubernamental }\end{array}$} & Media alta & 95 & 901 \\
\cline { 2 - 4 } & Media baja & 135 & 728 \\
\cline { 2 - 4 } & Baja & 104 & 772 \\
\hline Nota: Elaboración propia a partir de: Centro de Estudios Públicos (2016a). \\
\hline
\end{tabular}

Se observó la calidad de cada categoría, la cual determina qué tan bien se encuentra representada cada una de ellas en el modelo de dos dimensiones. Aquí, los valores cercanos a 1.000 indican que hay una mejor representación de esa categoría en el mapa perceptual. 
Así, las categorías con una mejor calidad, considerando aquellos valores que superan los 800, son: a) Posición política: centro, centroizquierda, izquierda y ninguna; y, b) Posición política: centro, centroizquierda, izquierda y ninguna. No obstante, la calidad más baja de las categorías consideradas en el modelo es de 631, para posición política: centroderecha, siendo este un valor que indica igualmente una buena calidad.

En cuanto a las masas, sus valores oscilan entre 5 y 210, siendo las siguientes categorías las que más pesan en el modelo de correspondencias: a) Posición política: ninguna; b) Confianza en el gobierno: nada de confianza y poca confianza; y c) Evaluación de la gestión gubernamental: baja y media baja. Así, estas categorías son las que obtienen un peso mayor en los perfiles fila y columna de modo que se encuentran mejor expresadas en el modelo.

El mapa perceptual que captura la información anteriormente expuesta se muestra a continuación:

Figura 1

Mapa perceptual del análisis de correspondencias múltiples

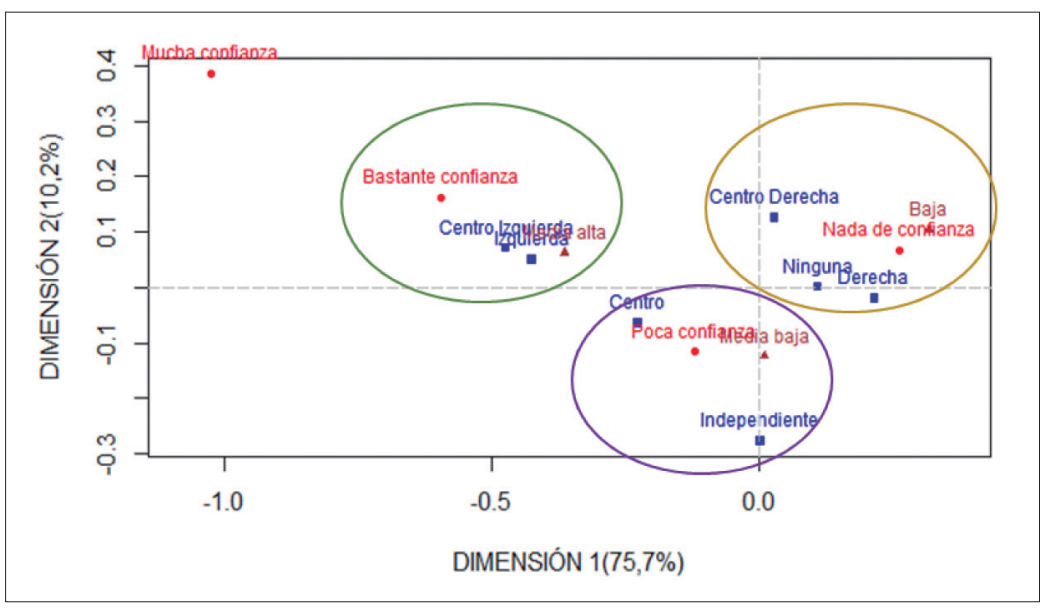

Nota: Elaboración propia a partir de: Centro de Estudios Públicos (2016a).

Como se observa en el mapa, la variable evaluación de la gestión gubernamental, además de que sus categorías presentan coefi- 
cientes aceptables, se encuentra distribuida de manera coherente con una polaridad definida, estando la evaluación media alta en el polo izquierdo del mapa, la categoría media baja en el centro y la categoría baja en el polo derecho. Por esto es que la primera dimensión se denominó evaluación de la gestión gubernamental, estableciéndose un continuo en donde las mejores evaluaciones se hallan a la izquierda y las peores a la derecha, sobre el eje horizontal.

Considerando que la variable confianza en el gobierno es la que mayor coherencia presenta en el mapa, a la vez que sus categorías poseen coeficientes aceptables, se define la segunda dimensión como confianza en el gobierno, estableciendo un continuo, en donde los mayores niveles de confianza se ubican hacia la parte superior del eje vertical, mientras que los niveles más bajos se presentan en las partes inferiores.

Como se observa en el mapa perceptual, se identificaron tres tipos de sujetos según la cercanía de los puntos. Las agrupaciones se formaron intentando priorizar la interpretación por cercanía dentro de cada uno de los cuatro cuadrantes. Por otro lado, cada grupo identificado se caracteriza por tener una evaluación de la gestión gubernamental, un nivel de confianza en el gobierno y una posición política específica.

El primer grupo identificado se encuentra en el cuadrante superior izquierdo. Se compone por los sujetos que evalúan la gestión gubernamental con notas medias altas, quienes tienen mayores probabilidades de adherir a una posición política de izquierda y centroizquierda, a la vez que son altas sus probabilidades de tener bastante confianza en el gobierno. Esto se condice con la hipótesis respecto a que los sujetos con una mejor evaluación de la gestión gubernamental tendrán posiciones políticas afines al gobierno izquierda y centroizquierda-, al tiempo que su confianza en el Poder Ejecutivo tiende a ser mayor. Sin embargo, debe considerarse que la evaluación de la gestión gubernamental no alcanza a ser alta, sino que se mantiene dentro de niveles medios, aunque es superior a la del resto de los grupos identificados. 
El segundo grupo identificado se encuentra en el cuadrante superior derecho. Se compone por los sujetos que evalúan la gestión gubernamental con notas bajas, quienes tienen mayores probabilidades de adherir a una posición política de derecha y centroderecha, o bien no sostener adhesión política alguna, a la vez que son altas sus probabilidades de tener nada de confianza en el gobierno. Esto se condice con la hipótesis respecto a que los sujetos con una mala evaluación de la gestión gubernamental tendrán posiciones políticas contrarias al gobierno - derecha y centroderecha-, al tiempo que su confianza en el Poder Ejecutivo tiende a ser baja.

A ello, se suma una cuestión interesante: sujetos sin adscripción política, fuera del continuo izquierda-derecha, también sostienen bajas evaluaciones de la gestión gubernamental y bajos niveles de confianza. Esta situación contradice las hipótesis propuestas sobre que la ausencia de posición política se relaciona con evaluaciones y confianza intermedia. Es probable que estos individuos asocien el espectro izquierdaderecha a la institucionalidad política, considerando que partidos y gobiernos se orientan y comunican según este continuo (Fuchs \& Klingemann, 1989). Así, al ser sujetos descontentos con el gobierno y que relacionan esta institución a los posicionamientos políticos tradicionales y tienden a reproducir su malestar en la ausencia de posicionamiento político en el continuo izquierda-derecha.

El tercer grupo identificado se encuentra en el cuadrante inferior izquierdo. Se compone por sujetos que evalúan la gestión gubernamental con notas medias bajas, quienes tienen mayores probabilidades de adherir a una posición política de centro o declararse independientes, a la vez que son altas sus probabilidades de tener poca confianza en el gobierno. Esto se condice con la hipótesis respecto a que sujetos con una evaluación media de la gestión gubernamental tendrán posiciones políticas de centro o independiente. Sin embargo, su evaluación de la gestión gubernamental es media tendiendo 
a baja, al tiempo que su confianza en el gobierno es reducida, aunque esta última está en un nivel superior a la del segundo grupo identificado. Por otro lado, los sujetos autodefinidos sin posición política no forman parte de este círculo, pues pertenecen al anteriormente descrito. En este sentido no se cumple la hipótesis a cabalidad.

Con todo, en términos generales, se comprueba que quienes mantienen posiciones políticas tendientes a la izquierda, afines al gobierno de Michelle Bachelet, suelen experimentar la situación gubernamental de forma positiva, otorgándole una buena evaluación al gobierno en su desempeño y depositándole altos niveles de confianza. En este sentido, como señala la literatura, tanto la confianza como evaluación dependerían de la relación entre la posición política de los individuos y la tendencia ideológica gubernamental (Bargsted \& Somma, 2013; Cárdenas et al., 2007; Kumlin, 2002; Zmerli, 2006).

\subsection{Relación entre evaluación de la gestión gubernamental e intención de asistir a votar}

A continuación, se presenta una tabla de contingencia que muestra los porcentajes columna entre intención de asistir a votar y evaluación de la gestión gubernamental —según los perfiles identificados en el análisis de conglomerados- con el objetivo de probar la idea de que los sujetos con buena evaluación de la gestión gubernamental se sienten incentivados a asistir a votar para respaldar a candidatos semejantes a los funcionarios de la administración bien evaluada (Arriagada et al., 2010; Morales, 2016; Morlino, 2007). 
Tabla 7

Porcentajes columna de evaluación de la gestión gubernamental - Intención de asistir a votar

\begin{tabular}{|l|c|c|c|c|}
\hline & \multicolumn{4}{|c|}{ Evaluación de la gestión gubernamental } \\
\hline Intención de asistir a votar & Baja & $\begin{array}{c}\text { Media } \\
\text { baja }\end{array}$ & $\begin{array}{c}\text { Media } \\
\text { alta }\end{array}$ & Total \\
\hline No, con toda seguridad & 15.26 & 11.51 & 12.20 & 12.87 \\
\hline Probablemente no & 7.63 & 12.34 & 10.71 & 10.42 \\
\hline Probablemente sí & 26.70 & 27.41 & 21.43 & 25.49 \\
\hline Sí, con toda seguridad & 50.41 & 48.75 & 55.66 & 51.23 \\
\hline Total & 100 & 100 & 100 & 100 \\
\hline Nota: Elaboración propia a partir de: Centro de Estudios Públicos (2016a). \\
\hline
\end{tabular}

Se observa que, efectivamente, la mayoría de los sujetos que evalúan la gestión gubernamental de forma media alta declaró que asistirá con toda seguridad a votar a las elecciones presidenciales de 2017 , siendo este un porcentaje de $55.66 \%$, bastante superior al resto de categorías de la variable intención de asistir a votar.

Sin embargo, tanto las categorías bajas y media baja de evaluación de la gestión gubernamental, tienen un porcentaje similar de sujetos que asistirá a votar con toda seguridad $-50.41 \%$ y $48.75 \%$, respectivamente. La misma situación se repite en torno a la categoría probablemente sí asistiré a votar, en donde los porcentajes de los tres perfiles de evaluación de la gestión gubernamental son similares, aunque los sujetos con evaluación media alta presentan un porcentaje inferior a quienes evalúan como baja y media baja la gestión gubernamental.

Así, no se cumple la hipótesis de que los sujetos con una evaluación alta de la gestión gubernamental tendrán una mayor intención de asistir a votar con el objetivo de respaldar a un gobierno similar del que tienen percepciones positivas. Por ello, pues no existe una diferencia importante entre la intención de asistir a votar y los distintos niveles de evaluación de la gestión gubernamental.

No obstante, debe considerarse que no se está analizando un grupo de sujetos que evalúe únicamente muy bien la gestión gu- 
bernamental, de modo que los resultados pueden verse afectados por ser personas con evaluaciones medias y bajas.

Además, la pregunta respecto a la intención de asistir a votar tiene una alta deseabilidad social, producto del deber cívico que aquello conlleva (Cumsille \& Nogales, 2014), de modo que la distribución de los encuestados puede no ser real y más bien estar constituida por lo que los sujetos consideran que es socialmente aceptado. Asimismo, los datos analizados fueron recogidos a un año de las elecciones, por lo que es posible que los sujetos no hayan podido anticipar su participación (Cumsille \& Nogales, 2014). Esto se constata en la participación electoral real, donde asistió a las urnas un $46.65 \%$ del padrón en la primera vuelta (SERVEL, 2019a), y un $48.98 \%$ en la segunda vuelta (SERVEL, 2019b), cuestión que se diferencia de los datos de la encuesta que anticipó un $76.72 \%$ de sujetos que probablemente asistirían a votar o lo harían con toda seguridad.

Por último, cabe señalar que no fue analizado el candidato específico por el que votarían los individuos, pues en el momento de la recolección de datos aún no existían candidatos oficialmente inscritos. Así, solo se analizó la relación entre la evaluación de la gestión gubernamental e intención de asistir a votar. Esto implica que, si bien se pueden aventurar conclusiones respecto a que las percepciones hacia el gobierno no inciden en la intención de asistir a votar, un análisis más adecuado se realizaría sobre la base de si los individuos que evalúan con notas altas al gobierno tenderían a mantener una intención de voto hacia candidatos del mismo sector. Para ello se requeriría analizar una base de datos que contemple los candidatos oficialmente inscritos para competir en los comicios.

\section{Conclusiones}

Retomando los resultados, se puede señalar que se cumple, en parte, la primera hipótesis del estudio, la cual proponía que se establecerían perfiles diferenciados por nivel de evaluación (baja, 
media y alta) en torno a la gestión gubernativa sobre el total de las áreas consideradas: delincuencia, crecimiento económico, salud, educación, transporte público, empleo, inflación, control de la corrupción, pobreza y pensiones. Sin embargo, no se constituye un perfil de evaluación alta, sino dos perfiles de evaluación media (media alta y media baja) y un tercero de evaluación baja.

La ausencia de perfiles de evaluación alta hacia el gobierno podría relacionarse con la existencia de un sentimiento de malestar generalizado que conllevaría a fenómenos de desafección, desaprobación y desconfianza con la política institucional (Joignant et al., 2016). La comprobación del modelo teórico que sostiene un descontento transversal, es decir, que no solo está asociado a quienes se oponen ideológicamente al gobierno de turno, tiene implicancias en la estabilidad democrática (Joignant et al., 2016), ante lo que se requerirían políticas de Estado que reduzcan el malestar, favoreciendo la calidad institucional.

Por otro lado, los resultados comprueban la segunda hipótesis propuesta en el artículo, pues aquellas personas que evalúan negativamente la gestión gubernamental tienen mayores probabilidades de reducir su confianza en el gobierno y de autodefinirse con posiciones políticas de derecha o centroderecha, contrarias a la administración de turno. Asimismo, aquellos que evalúan mejor la gestión gubernamental tienen mayores probabilidades de confiar en el gobierno, así como de pertenecer a posiciones políticas de izquierda o centroizquierda, concordantes con la administración de Michelle Bachelet. Finalmente, quienes evalúan la gestión con notas medias bajas y se definen como personas de centro o independientes, tienden a confiar medianamente en el gobierno.

Los únicos sujetos que no concuerdan con la hipótesis propuesta son quienes no adhieren a ninguna posición política del continuo izquierda-derecha, cuyos resultados muestran que tienen una baja confianza en el gobierno y negativa evaluación de la gestión gubernamental. Como se señaló, lo anterior podría relacionarse con que estos individuos asocian la institucionalidad 
con los conceptos de izquierda y derecha, por lo que la ausencia de autoposicionamiento político podría ser una extensión de su descontento, tal como lo sería su reducida confianza y negativa evaluación del gobierno.

Los resultados obtenidos se condicen con lo propuesto en el problema de investigación. Así como señala Morales (2008), la confianza sería una forma de apoyo al régimen político en función del desempeño de este. A ello se suma la perspectiva de Segovia et al. (2008), quienes argumentan que la confianza referiría a la evaluación que realizan los ciudadanos de las capacidades que tiene el gobierno. De esta manera, los resultados comprueban lo mencionado por los autores.

Por otro lado, algunos autores argumentan que la confianza en el gobierno se relaciona con la posición política de los sujetos (Cárdenas et al., 2007; Kumlin, 2002; Zmerli, 2006). Ante ello, se propuso como hipótesis que quienes adhieren a posiciones políticas cercanas al gobierno de turno, tenderían a confiar más en él, al tiempo que lo evaluarían con mejores calificaciones. Desde esta óptica, se observó que efectivamente esta relación se cumple en la dirección propuesta por los autores.

Además, se constató que no existe una mayor intención de asistir a elecciones por parte de quienes confieren una mejor evaluación a la gestión gubernamental, de modo que los ciudadanos no se ven incentivados a sufragar producto de sus positivas percepciones sobre la institucionalidad. Así, los resultados contradicen la tercera hipótesis, sustentada en lo propuesto por Morlino (2007), donde una buena gestión tendría consecuencias positivas en la sucesión del gobierno, pues los sujetos tendrían una motivación mayor para asistir a votar por candidatos afines a la administración anterior.

No obstante, con el fin de verificar esto con mayor precisión, futuras investigaciones debieran analizar la relación entre evaluación de la gestión gubernamental y la intención de votar por un candidato específico con el objetivo de descartar una relación entre evaluaciones positivas del gobierno y la intención de votar por un 
candidato del mismo sector político. Esto podría sustentar la idea del votante irracional, estrechamente relacionada con la desafección y desinformación política ciudadana (Achen \& Bartels, 2016). Así, no basta con analizar solamente el deseo de asistir a las urnas para descartar la hipótesis propuesta por Morlino (2007).

Con todo, cabe preguntarse cuánto influyen en la movilización de votantes los problemas de gestión y administración. En torno a esta interrogante, los resultados exploratorios arrojados por esta investigación parecen sugerir que su influencia es menor, apuntando hacia otros fenómenos de la política, tales como las apuestas programáticas de los candidatos e, incluso, la posición política y confianza analizadas en el presente estudio, entre muchos otros factores.

Finalmente, la cuestión que subyace a las conclusiones obtenidas es cómo se relacionan las actitudes políticas en un contexto democrático. A ello, los resultados aportan que, de manera general, la posición política parece seguir teniendo relevancia a la hora de evaluar la gestión gubernamental y depositar confianza en el gobierno, al tiempo que estos dos últimos ámbitos están estrechamente ligados. En contraposición, la participación electoral no parece relacionarse con los juicios que los sujetos hacen sobre los gobiernos de turno.

Sin embargo, una futura investigación orientada en esta línea, así como la lectura de los resultados de este estudio, debe realizarse considerando las condiciones contextuales en Chile, en tanto la evaluación de los gobiernos en general es negativa y, al mismo tiempo, los niveles de participación electoral son bajos.

\section{Referencias}

Achen, C. \& Bartels, L. (2016). Democracy for realists. Why elections do not produce responsive government. Woodstock: Princeton University Press.

Arriagada, A., Navia, P. \& Schuster, M. (2010). ¿Consumo luego pienso, o pienso y luego consumo? Consumo de medios, pre- 
disposición política, percepción económica y aprobación presidencial en Chile. Revista de Ciencia Política, 30(3), 669-695. Disponible en: http://dx.doi.org/10.4067/S0718090X2010000300005

Bargsted, M. A. \& Somma, N. M. (2013). Social cleavages and political dealignment in contemporary Chile, 1995-2009. Party Politics, 22(1), 1-20. Disponible en: https://doi. org $/ 10.1177 / 1354068813514865$

Barozet, E. (2016). Entre la urna, las redes sociales y la calle: las relaciones entre movimientos sociales y partidos políticos en el Chile democrático. En M. A. Garretón (Ed.), La gran ruptura. Institucionalidad politica y actores sociales en el Chile del siglo XXI (pp. 21-58). Santiago de Chile: LOM Ediciones.

Cárdenas, M., Parra, L., Picón, J., Pineda, H. \& Rojas, R. (2007). Las representaciones sociales de la política y la democracia. Última Década, 15(26), 53-78. Disponible en: http://dx.doi. org/10.4067/S0718-22362007000100004

Cea, M. A. (2004). Análisis multivariante. Teoría y práctica de la investigación social. Madrid: Síntesis.

Centro de Estudios Públicos. (2016a). Base de Datos. (Encuesta CEP N ${ }^{\circ}$ 78. Estudio Nacional de Opinión Pública $\mathrm{N}^{\circ} 48$ - Tercera Serie. Noviembre-diciembre 2016). Santiago de Chile: Centro de Estudios Públicos.

Centro de Estudios Públicos. (2016b). Manual del Usuario. (Encuesta CEP $\mathrm{N}^{\circ}$ 78. Estudio Nacional de Opinión Pública $\mathrm{N}^{\circ} 48$ - Tercera Serie. Noviembre-diciembre 2016). Santiago de Chile: Centro de Estudios Públicos.

Cumsille, G. \& Nogales, A. (2014). Criterios para la formulación de marcos muestrales en encuestas electorales para escenarios de voto voluntario: Análisis de modelo experimental. Trabajo presentado en el VI Congreso Latinoamericano de Opinión Pública, WAPOR, Santiago de Chile.

Foster, C. B. (1984). The performance of rational voter models in recent presidential elections. The American Political Science Review, 78(3), 678-690. Disponible en: https://doi. org $/ 10.2307 / 1961836$

Fuchs, D. \& Klingemann, H. D. (1989). The left-right schema. En M. K. Jennings, J. van Deth, S. H. Barnes, D. Fuchs, F. J. Heunks, 
R. Inglehart... J. J. Thomasse (Eds.), Continuities in political action (pp. 203-234). Berlín: Walter de Gruyter.

Garretón, M. A. (2016). La ruptura entre política y sociedad. Una introducción. En M. A. Garretón (Ed.), La gran ruptura. Institucionalidad politica y actores sociales en el Chile del siglo XXI (pp. 11-19). Santiago de Chile: LOM Ediciones.

Greenacre, M. (2008). La práctica del análisis de correspondencias. Madrid: Fundación BBVA.

Inglehart, R. \& Klingemann, H. D. (1979). Ideological conceptualization and value priorities. En S. H. Barnes, M. Kaase, K. R. Allerbeck, B. G. Farah, F. Heunks, R. Inglehart \& L. Rosenmayr (Eds.), Political action. Mass participation in five western democracies (pp. 203-213). Beverly Hills: Sage Publications.

Joignant, A., Morales, M. \& Fuentes, C. (2016). Malaise in representation: Attitudes, beliefs, behaviors and causalities. En A. Joignant, M. Morales \& C. Fuentes (Eds.), Malaise in representation in Latin American countries. Chile, Argentina, and Uruguay (pp. 1-43). New York: Palgrave Macmillan.

Kumlin, S. (2002). The personal and the political: how personal welfare state experiences affect political trust and ideology. Gothenburg: Department of Political Science, University of Gothenburg.

Meléndez, C. \& Rovira, C. (2017). Political identities: The missing link in the study of populism. Party Politics, 25(4), 520-533. Disponible en: https: / / doi.org/10.1177/1354068817741287

Montero, J. R., Zmerli, S. \& Newton, K. (2008). Confianza social, confianza política y satisfacción con la democracia. Reis, (122), 11-54. Disponible en: http:/ / www.reis.cis.es/REIS/PDF/ REIS_122_011238570387245.pdf

Morales, M. (2008). Evaluando la confianza institucional en Chile. Una mirada desde los resultados LAPOP. Revista de Ciencia Política, 28(2), 161-186. Disponible en: http://dx.doi. org/10.4067/S0718-090X2008000200007

Morales, M. (2016). Tipos de identificación partidaria. América Latina en perspectiva comparada, 2004-2012. Revista Estudios Sociales, (57), 25-42. Disponible en: http:/ /dx.doi.org/10.7440/ res57.2016.02

Morlino, L. (2007). Explicar la calidad democrática: ¿Qué tan relevantes son las tradiciones autoritarias? Revista de Ciencia Polí- 
tica, 27(2), 3-22. Disponible en: http:/ / dx.doi.org/10.4067/ S0718-090X2007000300001

Newton, K. \& Norris, P. (septiembre, 1999). Confidence in public institutions: Faith, culture or performance? Trabajo presentado en la Annual Meeting of the American Political Science Association, Atlanta.

Ruiz, C. (2016). Crisis política en Chile: Neoliberalismo, cambios sociales y democracia. En M. A. Garretón (Ed.), La gran ruptura. Institucionalidad política y actores sociales en el Chile del siglo XXI (pp. 83-108). Santiago de Chile: LOM Ediciones.

Segovia, C. (2016). Malaise and democracy in Chile. En A. Joignant, M. Morales \& C. Fuentes (Eds.), Malaise in representation in Latin American countries. Chile, Argentina, and Uruguay (pp. 6992). New York: Palgrave Macmillan.

Segovia, C., Haye, A., González, R., Manzi, J. \& Carvacho, H. (2008). Confianza en instituciones políticas en Chile: Un modelo de los componentes centrales de juicios de confianza. Revista de Ciencia Política, 28(2), 39-60. Disponible en: http:/ / dx.doi.org/10.4067/S0718-090X2008000200002

Servicio Electoral de Chile. (2, diciembre, 2019a). Estadísticas de participación a nivel nacional Elecciones 2017: Servicio Electoral de Chile. Recuperado el 2 de diciembre de 2019 de: https://www. servel.cl/estadisticas-de-participacion-a-nivel-nacional/

Servicio Electoral de Chile. (2, diciembre, 2019b). Estadísticas de participación a nivel nacional Segunda Votación de la Elección Presidencial: Servicio Electoral de Chile. Recuperado el 2 de diciembre de 2019 de: https:/ / www.servel.cl/estadisticasde-participacion-a-nivel-nacional-segunda-votacion-de-laeleccion-presidencial/

Zmerli, S. (julio, 2006). Political confidence in Europe. Trabajo presentado en 20th IPSA World Congress, Fukuoka, Japón. 